\title{
Association of airflow limitation with trauma exposure and post-traumatic stress disorder
}

\author{
C. Spitzer*, B. Koch" ${ }^{\#}$, H.J. Grabe ${ }^{\Uparrow}$, R. Ewert", S. Barnow ${ }^{+}$, S.B. Felix ${ }^{\#}$, T. Ittermann ${ }^{\S}$, \\ A. Obst ${ }^{\#}$, H. Völzke ${ }^{\S}$, S. Gläser ${ }^{\#}$ and C. Schäper ${ }^{\#}$
}

ABSTRACT: Trauma exposure and post-traumatic stress disorder (PTSD) are associated with self-reported asthma and chronic obstructive pulmonary disease. However, these conditions have not yet been related to objective measures of lung function.

1,772 adults from the general population were assessed regarding their medical histories and spirometric lung function. Additionally, they were administered a PTSD interview, and assigned to three groups: no trauma; trauma, but no PTSD; and trauma with PTSD.

Adjusting for sociodemographic, clinical and lifestyle factors, subjects with PTSD had significantly higher odds ratios for most asthma-related symptoms than PTSD-negative participants (OR 3.2-8.8). The mean ratio of forced expiratory volume in $1 \mathrm{~s}$ (FEV1) to forced vital capacity (FVC) was lowest in the PTSD group and highest in those without trauma exposure. Traumatic stress was independently associated with FEV1 and FEV $1 / F V C$. Participants with PTSD, compared with those without, had a significantly increased risk for airflow limitation independent of its definition (OR 4.2-7.8).

This is the first study relating traumatic stress and PTSD, respectively, to objective parameters of lung function. Our findings suggest an association of trauma exposure and PTSD with airflow limitation, which may be mediated by inflammatory processes.

KEYWORDS: Airflow limitation, asthma, chronic obstructive pulmonary diseases, post-traumatic stress disorder, pulmonary function testing, trauma

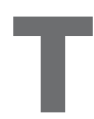
here is growing evidence for a close association between exposure to traumatic stress, e.g. childhood maltreatment or combat experience, and poor physical health, particularly pulmonary diseases such as asthma and chronic bronchitis [1-3]. For instance, studies of adult general population samples found a graded relationship between the numbers of childhood adversities and the risk for chronic obstructive pulmonary disease (COPD) [4-6] and asthma [7]. Likewise, traumatic experiences in adulthood are related to the development or worsening of asthma, as indicated by research among adults exposed to the September 11, 2001 World Trade Center terrorist attack [8-10].

Post-traumatic stress disorder (PTSD), as the most common psychological sequela of traumatic experiences, has also been linked to respiratory dysfunction [11-15]. For example, large community studies found that subjects with PTSD had a three times higher risk for asthma and COPD than those without PTSD [3, 11]. Similar results were found in primary care patients [16]. Likewise, PTSD-positive male combat veterans had an increased risk to report chronic pulmonary diseases compared with those who were PTSDnegative [15]. Among female veterans, those with PTSD had a 1.6-fold higher risk for self-reported asthma than those without PTSD [13]. Another veteran study indicated that the strongest association between PTSD and other diseases, including cardiovascular and digestive diseases, identified asthma [14]. Finally, the association between PTSD symptoms and asthma remained after adjustment for familial, genetic and other confounders in a twin study [17].

In summary, consistent findings strongly suggest an association of trauma and PTSD with respiratory conditions characterised by airflow limitation. However, this relationship might be compromised

\section{AFFILIATIONS}

${ }^{*}$ Dept of Psychosomatic Medicine and Psychotherapy, University Medical Center Hamburg-Eppendorf and Schön Klinik Hamburg-Eilbek, Hamburg,

\#Dept of Internal Medicine B Cardiology, Pulmonary Medicine, Infectious Diseases and Intensive Care Medicine,

"Dept of Psychiatry and

Psychotherapy,

${ }^{\S}$ Institute for Community Medicine, Ernst-Moritz-Arndt-University,

Greifswald, and

${ }^{+}$Institute of Psychology, RuprechtKarls University, Heidelberg, Germany.

CORRESPONDENCE

C. Spitzer

Dept of Psychosomatic Medicine and Psychotherapy

University Medical Center HamburgEppendorf and Schön Klinik Hamburg-Eilbek Martinistr. 52 20246 Hamburg Germany

E-mail: c.spitzer@uke

uni-hamburg.de

Received:

Feb 192010

Accepted after revision:

Aug 112010

First published online:

Aug 202010 
by several methodological problems. First, the diagnoses of asthma or COPD were ascertained by self-report, but not corroborated by medical records or physicians' assessment. Due to the use of self-report of respiratory disorders, it remains unknown whether the observed association is solely the result of self-report bias, particularly as asthmatic and PTSD-positive subjects were found to inaccurately perceive and report symptoms and distress, possibly due to negative emotional states, such as anxiety and depression, which are often associated with both conditions [18-20]. Secondly, asthma-related symptoms resemble certain features of panic attacks (e.g. chest tightness and shortness of breath), making it difficult to distinguish between these two conditions. Correspondingly, male combat veterans with PTSD did not have more commonly physician-diagnosed pulmonary diseases than those without PTSD [21], and the agreement between patients' and physicians' reports of respiratory dysfunction was low in Vietnam veterans independent of their PTSD status [22]. Thirdly, traumatic experiences and PTSD, respectively, have not yet been related to objective measures of lung function. Finally, many of the aforementioned investigations focused on either specific traumatic events or selected populations, e.g. childhood maltreatment $[4,6]$ or combat veterans, respectively [13-15, 17, 23], making it difficult to compare findings across various studies and raising the question of generalisability. Thus, general population studies, which include a wide variety of traumatic experiences, are important. In light of these considerations, the objective of our study was two-fold: 1) to investigate the differential relationship of traumatic stress and PTSD with selfreported symptoms and conditions of pulmonary disease in a general population sample, controlling for potential confounders; and 2) to assess the association between trauma exposure, PTSD and objective measures of lung function, i.e. forced expiratory volume in $1 \mathrm{~s}$ (FEV1), forced vital capacity (FVC) and FEV1/FVC. Additionally, traumatic stress and PTSD were related to airflow limitation as determined by spirometry. However, because the prevalence of airflow limitation strongly depends on its definition, which is currently under intense debate [24], we applied different operationalisations [25-27].

\section{METHODS}

\section{Procedure and subjects}

Participants were recruited as part of the Study of Health in Pomerania (SHIP), which is an ongoing population-based project in north-eastern Germany involving the three cities of Greifswald, Stralsund and Anklam, and 29 surrounding communities. The population density of this predominantly rural area is low and ranges $50-1,485$ inhabitants $\cdot \mathrm{km}^{-2}$. From the total population of 212,157 inhabitants, a representative sample of 7,008 persons aged 20-79 yrs was selected from population registries, considering the inclusion criteria of German citizenship and residency in West Pomerania. In Germany, each inhabitant is obliged to be registered. Information on name, birthday and residence address is collected from the day of birth onwards. Any relocation must be declared. These data base can be used for scientific purposes, and the completeness of information allows drawing population samples with a high grade of representativeness. The twostage cluster sampling method was adopted from the World Health Organization (WHO) Multinational Monitoring of Trends and Determinants in Cardiovascular Disease (MONICA) project in Augsburg, Germany, and yielded 12 5-yr age strata for both sexes, each including 292 individuals [28]. A total of 4,310 individuals (2,193 females), $68.8 \%$ of all eligible subjects, took part in the baseline study (SHIP-0), which was performed between October 1997 and May 2001. The study was approved by the local ethics committee (Ethikkommission an der Medizinischen Fakultät, Institut für Pharmakologie, Ernst-Moritz-Arndt-Universität Greifswald, Greifswald, Germany). The detailed objectives and design of SHIP are published elsewhere [29]. In brief, data were collected in two medical centres specifically established for this study and located within the local hospitals. Participants were offered free transportation to and from the examination centres, a meal and $€ 15$ as incentives. The data collection comprised four parts: a health- and risk factor-related self-report questionnaire, an oral health examination, a medical examination and a computer-assisted health-related interview. The latter was conducted by trained interviewers; additionally, there was a continuous quality monitoring [29].

The present study was part of the first 5-yr follow-up investigation (SHIP-I) performed between December 2002 and December 2006 [30], i.e. a cross-sectional study nested in a long-term community-based cohort study. For SHIP-I, there were 130 passive nonresponders due to migration and 231 deceased subjects. Of the remaining 3,949 eligible persons, 649 were active nonresponders. Thus, a total of 3,300 participants of the original study were followed up ( $83.6 \%$ response rate). The nonresponders were significantly older, more often single, less educated and unemployed [31]. Pulmonary function testing was offered to any subject volunteering in SHIP-I, and was finally carried out on a random subsample comprising 1,809 subjects [32, 33]. Those performing spirometry were younger, less often separated, divorced or widowed, had a higher educational level, were less often current smokers, and had lower frequencies of trauma exposure and PTSD than nonperformers (results not presented in detail). Of the 1,809 participants with spirometry data, 37 subjects had to be excluded for the following reasons: one $(0.05 \%)$ individual did not complete the interview, another $23(1.3 \%)$ individuals exhibited cognitive impairment as defined by a Mini Mental State Examination (MMSE) score of $\leqslant 23$, one $(0.05 \%)$ subject suffered from lung cancer, and nine $(0.5 \%)$ subjects had incomplete pulmonary function testing data. Thus, 1,772 adults living in the community were analysed in the present study.

\section{Psychological assessment}

The health-related interview of SHIP-I included the PTSD module of the Structured Clinical Interview for DSM-IV (SCID) [34], which is frequently administered by traumatic stress professionals [35], the Composite International Diagnostic Screener (CID-S) [36], and the MMSE [37]. The PTSD interview begins by directly asking about the exposure to events included as traumas in the Diagnostic and Statistical Manual (DSM)-IV (criterion A1). If a participant answered "no" to each of the trauma questions, the module was terminated. Otherwise, the interview was continued to assess the DSMIV PTSD symptoms, including fear, helplessness or horror as the initial reaction (criterion A2), five re-experiencing symptoms (criterion B), seven avoidance symptoms (criterion C), and five arousal symptoms (criterion D). If participants did not pass the required diagnostic threshold, the interview was 
terminated. Because there is evidence for a graded relationship between the number of adverse childhood experiences and the risk of obstructive airway disease [38], traumatic stress was not only treated as binary variable (0: absent; 1 : present), but was also defined by the number of traumatic events meeting both DSM-IV A1 and A2 criteria.

The CID-S [36] was used to estimate the 12-month prevalence rate of panic attacks, anxiety and depression [27]. It comprises items reflecting the so-called stem questions of the Composite International Diagnostic Interview assessing the core symptoms of the respective disorders. Panic attacks were classified when subjects endorsed the corresponding item. Endorsement of at least one out of six items reflecting anxiety and depression was used as proxy measure of negative emotional states. The sensitivity of the CID-S was reported to be $85.3 \%$ [36].

Cognitive functioning was assessed by means of the MMSE [37] before administering the other measures. MMSE scores of $\leqslant 23$ were considered to indicate cognitive impairment, and participants not exceeding this cut-off were excluded.

\section{Medical history of lung disease and pulmonary function testing}

Personal medical history was assessed by a computer-assisted personal interview, including several asthma-related questions, modified from the interview applied within the European Community Respiratory Health Survey [39]. Finally, respondents were also asked if they had suffered from a physician-diagnosed chronic bronchitis or asthma in the year prior to the study. Medication was recorded according to the Anatomical Therapeutic Chemical (ATC) classification [40] and drugs of interest (ATC code R03) were treated as binary variables (0: no use; 1: use). Participants also underwent routine medical examination including anthropometric measurements to assess height and weight. Smoking status was classified as never smoking (0), former smoking (1) or current smoking (i.e. one or more cigarettes per day; 2). Marital status was subdivided into three categories: never married (0), married (1), and divorced, separated or widowed (2). Corresponding to the German school system, education was treated as dichotomous variable $(0: \leqslant 10 \mathrm{yrs} ; 1: \geqslant 11 \mathrm{yrs})$.

Pulmonary function testing was conducted using a body plethysmograph equipped with a pneumotachograph (VIASYS Healthcare, JAEGER, Hoechberg, Germany) that meets the American Thoracic Society criteria [41, 42]. The volume signal of the equipment was calibrated with a 3.0-L syringe connected to the pneumotachograph in accordance with the manufacturer's recommendations, and this was performed at least once on each testing day. Barometric pressure, temperature and relative humidity were recorded every morning. Calibration of the volume was examined under ambient temperature and pressure conditions, and the integrated volumes were body temperature pressure saturated corrected [41, 42]. The participants performed at least three lung function manoeuvres in order to obtain a minimum of two acceptable and reproducible values [26]. Immediate on-screen error codes indicating the major acceptability (including start, minimal duration and end of test) and reproducibility criteria supported the attempt for standardised procedures. Prior to the tests, the required manoeuvres were demonstrated by the operator, and the individuals were encouraged and supervised throughout the performance of the tests. The best results for FVC and FEV1 were taken. We also calculated \% predicted values of FEV1 and FVC, based on the sex-specific equations of the European Community for Coal and Steel (ECCS) [26] and of the Third National Health and Nutrition Examination Survey (NHANES III) [27]. Additionally, three definitions of airflow limitation were applied: 1) a fixed ratio of $\mathrm{FEV} 1 / \mathrm{FVC} \leqslant 70 \%$ according to the recommendation of the Global Initiative for Chronic Obstructive Lung Disease [25]; 2) FEV1/FVC values below the 5th percentile according to the equation of the ECCS [26]; and 3) FEV1/FVC values below the 5th percentile derived from the equation of the NHANES III [27].

\section{Statistical analysis}

All analyses were computed using the Statistical Package for the Social Sciences (SPSS, version 14.0; SPSS Inc., Chicago, IL, USA). We applied the Chi-squared test for categorical variables and ANOVA for continuous variables, followed by post hoc pairwise comparisons according to the method of Bonferroni. In order to determine the relationship of trauma and PTSD with self-reported respiratory symptoms and conditions, we performed logistic regression analyses with these binary variables (present versus absent) as the dependent variable, and trauma exposure and PTSD as independent variables; sex, age, height, marital status, education, smoking, panic attacks and negative emotional states were considered as potential confounders. The differential impact of traumatic stress and PTSD on lung function parameters were analysed by linear regressions with absolute and \% predicted values of FEV1, FVC and FEV1/FVC as dependent variables. Significance level was set at $\mathrm{p}<0.05$.

\section{RESULTS}

The study population comprised 909 (51.3\%) females and 863 $(48.7 \%)$ males with a mean \pm SD age of $52.1 \pm 13.6$ yrs (range $25-$ $86 \mathrm{yrs})$. Of the 1,772 community residents included in the present study, 915 (51.6\%) subjects had been exposed to at least one traumatic event. 28 participants met criteria for PTSD (1.6\% of the total study population and $3.6 \%$ of those with trauma exposure). Participants were assigned to one of the following groups: no trauma exposure (no trauma; $n=857$ ); trauma, but no PTSD (trauma; $\mathrm{n}=887$ ); and trauma with the development of PTSD (PTSD; $n=28$ ). These three subsamples differed significantly with respect to age, marital status, the frequency of panic attacks and negative emotional states, and height (table 1).

Associations between trauma, PTSD and asthma-related variables are shown in table 2. PTSD-positive participants reported the highest proportions, followed by those with trauma exposure but without PTSD; those without traumatic experiences had the lowest proportions. Traumatised subjects had an increased likelihood of reporting awakening due to shortness of breath and an attack of coughing, compared with participants without trauma exposure. Compared with subjects without PTSD, the PTSD group had a significantly higher risk for almost all asthma-related symptoms.

Between-group comparisons revealed that nontraumatised participants had significantly higher values for both FEV1 and FVC than the two other groups, which did not differ from 
TABLE 1 Sociodemographic and clinical characteristics of the study population

\begin{tabular}{|c|c|c|c|c|c|}
\hline Subjects n & 857 & 887 & 28 & & \\
\hline Age yrs $^{\#}$ & $49.3 \pm 12.4$ & $54.8 \pm 14.0$ & $54.8 \pm 16.2$ & 39.207 & $\leqslant 0.001$ \\
\hline Marital status \% & & & & 24.446 & $\leqslant 0.001$ \\
\hline Never married & 16.8 & 13.2 & 10.7 & & \\
\hline School education \% & & & & 3.521 & $\leqslant 0.172$ \\
\hline$\leqslant 10 \mathrm{yrs}$ & 82.6 & 80.6 & 92.9 & & \\
\hline$\geqslant 11 \mathrm{yrs}$ & 17.4 & 19.4 & 7.1 & & \\
\hline Smoking status \% & & & & 3.335 & $\leqslant 0.503$ \\
\hline Never & 43.7 & 44.1 & 32.1 & & \\
\hline Height $\mathbf{c m}^{\pi}$ & $170.5 \pm 9.0$ & $169.1 \pm 9.1$ & $166.9 \pm 9.4$ & 6.165 & $\leqslant 0.002$ \\
\hline Weight kg & $80.2+15.8$ & $80.4+15.9$ & $79.9+16.2$ & 0.029 & $\leqslant 0.972$ \\
\hline
\end{tabular}

Data are presented as mean \pm SD, unless otherwise stated. PTSD: post-traumatic stress disorder. ${ }^{*}$ : post hoc pairwise comparisons (Bonferroni) indicated that traumatised subjects were significantly older than nontraumatised participants; ${ }^{\circ}$ : post hoc pairwise comparisons (Bonferroni) indicated that traumatised subjects were significantly shorter than nontraumatised participants but PTSD positive subjects did not differ from the two other groups.

each other (table 3). The FEV1/FVC ratio was significantly lower in traumatised subjects compared with the group of nontraumatised participants, while those with PTSD did not differ from the two other groups. There were no significant differences in the \% predicted values of FEV1 and FVC based on the ECCS equations between the groups. With respect to $\%$ predicted values according to the NHANES III equation, PTSD-positive participants had significantly lower FEV1 values than the two other groups, and traumatised subjects had significantly lower FVC values than nontraumatised ones, while the PTSD group did not differ from the others. When defining airflow limitation according to the ECCS criteria or $\mathrm{FEV} 1 / \mathrm{FVC} \leqslant 70 \%$, the PTSD group had a significantly increased risk for airflow limitation compared with residents without PTSD (OR 7.8, 95\% CI 1.8-33.2 and OR 4.2, 95\% CI 1.0-17.4, respectively). Traumatised participants did not have a higher risk for airflow limitation than those without trauma exposure.

TABLE 2 Association between self-reported respiratory symptoms and conditions, trauma exposure and post-traumatic stress disorder (PTSD)

\begin{tabular}{|c|c|c|c|c|c|c|c|}
\hline & \multirow[t]{2}{*}{ No trauma } & \multirow[t]{2}{*}{ Trauma } & \multirow[t]{2}{*}{ PTSD } & \multirow[t]{2}{*}{ Chi-squared } & \multirow[t]{2}{*}{ p-value } & \multicolumn{2}{|c|}{$\mathrm{OR}^{\#}(95 \% \mathrm{Cl})$} \\
\hline & & & & & & Trauma & PTSD \\
\hline Subjects $n$ & 857 & 887 & 28 & & & & \\
\hline \multicolumn{8}{|c|}{$\begin{array}{l}\text { Self-reported respiratory symptoms and } \\
\text { conditions } \%\end{array}$} \\
\hline Woken with shortness of breath & 2.0 & 2.9 & 25.0 & 52.461 & 0.001 & $1.5(1.1-1.8)^{\star \star}$ & $4.9(1.8-13.8)^{\star \star}$ \\
\hline Woken by an attack of coughing & 5.8 & 9.2 & 39.3 & 44.454 & 0.001 & $1.2(1.0-1.4)^{\star}$ & $3.7(1.6-8.5)^{\star \star}$ \\
\hline Asthma attack & 0.7 & 1.2 & 14.3 & 42.780 & 0.001 & $1.2(0.8-1.8)$ & $8.8(2.4-32.5)^{\star \star \star}$ \\
\hline Medication & 2.8 & 5.9 & 17.9 & 20.881 & 0.001 & $1.2(1.0-1.5)^{+}$ & $3.6(1.2-10.5)^{\star}$ \\
\hline Asthma & 1.6 & 3.2 & 10.7 & 11.767 & 0.003 & $1.2(0.9-1.5)$ & $3.2(0.9-11.9)^{*}$ \\
\hline Chronic bronchitis & 3.7 & 5.0 & 17.9 & 13.016 & 0.001 & $1.1(0.9-1.3)$ & $2.6(0.9-7.7)^{+}$ \\
\hline
\end{tabular}

\#: adjusted for age, sex, height, marital status, education, smoking, panic attacks and negative emotional states; ": anatomical therapeutical chemical classification code $R 03 ;{ }^{+}: p \leqslant 0.10 ; *^{*}: p \leqslant 0.05 ; * *: p \leqslant 0.01 ; * * * p \leqslant 0.001$ 


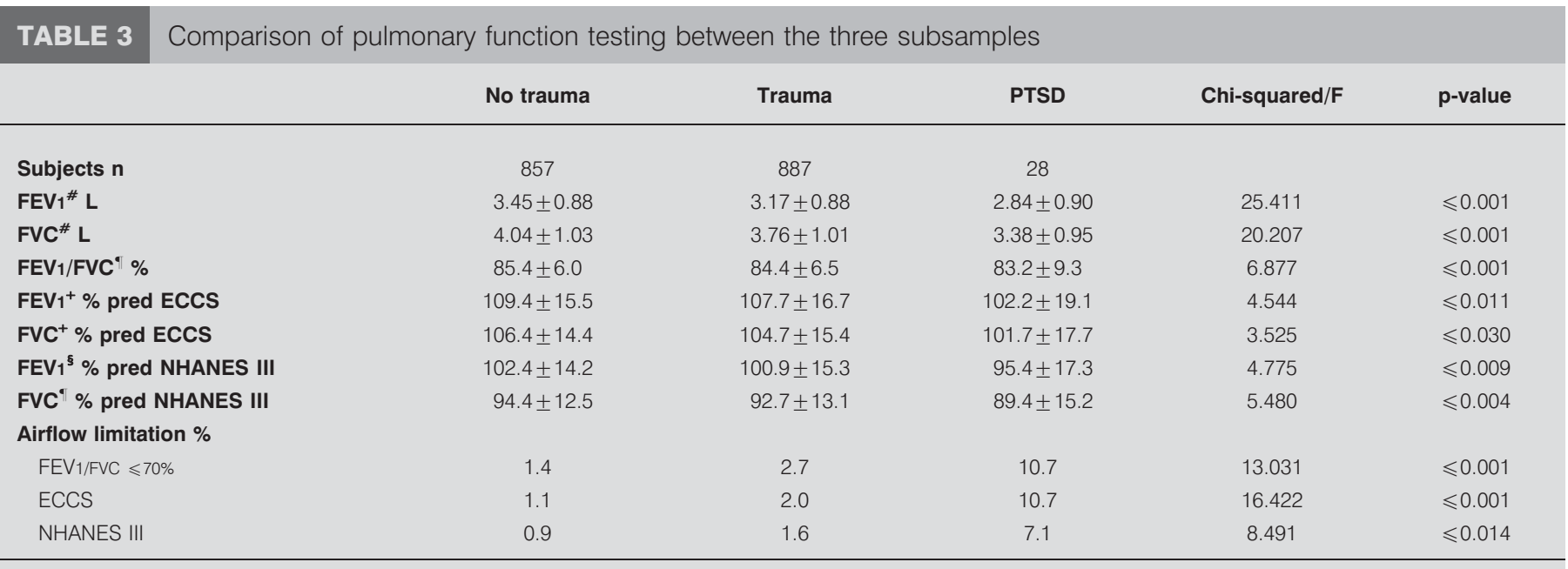

Data are presented as mean \pm SD, unless otherwise stated. PTSD: post-traumatic stress disorder; FEV1: forced expiratory volume in 1 s; FVC: forced vital capacity; \% pred: \% predicted; ECCS: European Community for Coal and Steel; NHANES III: Third National Health and Nutrition Examination Survey. ${ }^{\#}$ : post hoc pairwise comparisons (Bonferroni) indicated that nontraumatised subjects had significantly higher values than the two other groups, which did not differ from each other; ": post hoc pairwise comparisons (Bonferroni) indicated that traumatised subjects had significantly lower values than nontraumatised participants but PTSD-positive subjects did not differ from the two other groups; ${ }^{+}$: post hoc pairwise comparisons (Bonferroni) did not reveal any group differences; ${ }^{\S}$ : post hoc pairwise comparisons (Bonferroni) indicated that PTSD-positive subjects had significantly lower predicted values than nontraumatised participants, who did not differ from the traumatised group.

To determine the associations of traumatic stress and PTSD with lung function independent of other determinants, we performed linear regressions with absolute and \% predicted values of FEV1, FVC and FEV1/FVC as dependent variables (table 4). Traumatic stress, as defined by the numbers of traumatic events, was significantly related to absolute FEV1 and FEV1/FVC; furthermore, it was associated with predicted values of FEV1 and FVC (using the ECCS equation) and with predicted FVC values (NHANES III). There were no associations of PTSD with these parameters. Reanalyses of the data treating traumatic stress as binary variable (absent versus present) did not yield substantially different results: PTSD was not associated with any measure of lung function; traumatic stress was related to the absolute value of FEV1 and the predicted values of FVC, but not to FEV1/FVC or predicted values of FEV1 (data not shown).

\section{DISCUSSION}

To the best of our knowledge, this is the first study relating traumatic stress and PTSD to objective parameters of pulmonary function testing, extending prior research suggesting an association between trauma, PTSD and self-reported pulmonary disease [3, 8-15]. We found that PTSD was much more strongly linked with almost all asthma-like symptoms than trauma exposure alone, which is consistent with other studies [3,11-15]. Pulmonary function testing revealed that nontraumatised participants had significantly higher FEV1 and FVC values than those with trauma exposure and PTSD, respectively. FEV1/FVC was significantly lower in the traumatised group compared to those without traumatic experiences and PTSD; the same was true for the predicted FVC values according to the NHANES III equation. Finally, PTSD-positive participants had significantly lower predicted FEV1 values according to the NHANES III equation than the two other groups that did not differ from each other. Regardless of its definition, airflow limitation was significantly more frequent in the PTSD group compared to those without PTSD, even when controlling for relevant confounders. However, PTSD was not independently related to lung function parameters, as revealed by linear regression analyses. In contrast, traumatic stress was associated with absolute values of FEV1 and predicted values of FVC.

Before discussing the possible mechanisms linking traumatic stress and PTSD with airflow limitation, it has to be emphasised that our results are puzzling in that they do not follow a consistent pattern. While PTSD, but not trauma exposure, was associated with airflow limitation when defined categorically, traumatic stress, but not PTSD, was related to dimensional measures of lung function indicating airway obstruction. These inconsistencies deserve some considerations. From a statistical point of view, categorisations of continuous variables should be avoided [43]. Thus, our approach using the dimensional measures of lung function as outcomes might be considered superior to the use of the dichotomous variable airflow limitation. However, a clear-cut distinction between normal and pathological values is preferred from a clinical perspective, suggesting that airflow limitation as an outcome is clinically more meaningful than dimensional spirometry values. Future studies will need to clarify which outcomes are adequate before it will be possible to resolve the controversial debate about the differential impact of traumatic stress and PTSD on physical health in general and airway obstruction in particular [3, 44, 45].

Several pathways to poor physical health have been suggested for both trauma exposure and PTSD, including biological changes, engagement in poor health behaviour, and dysfunctional coping [1, 14, 45, 46]. All of these lead to increased allostatic load, defined as the cumulative burden that an organism experiences due to repeated cycles of adaptation, eventually causing somatic changes that promote disease [47]. 
TABLE 4 Differential association of trauma exposure and post-traumatic stress disorder (PTSD) with absolute and \% predicted values of forced expiratory volume in $1 \mathrm{~s}(\mathrm{FEV} 1)$, forced vital capacity $(\mathrm{FVC})$ and $\mathrm{FEV} 1 / \mathrm{FVC}$ in linear regression analyses

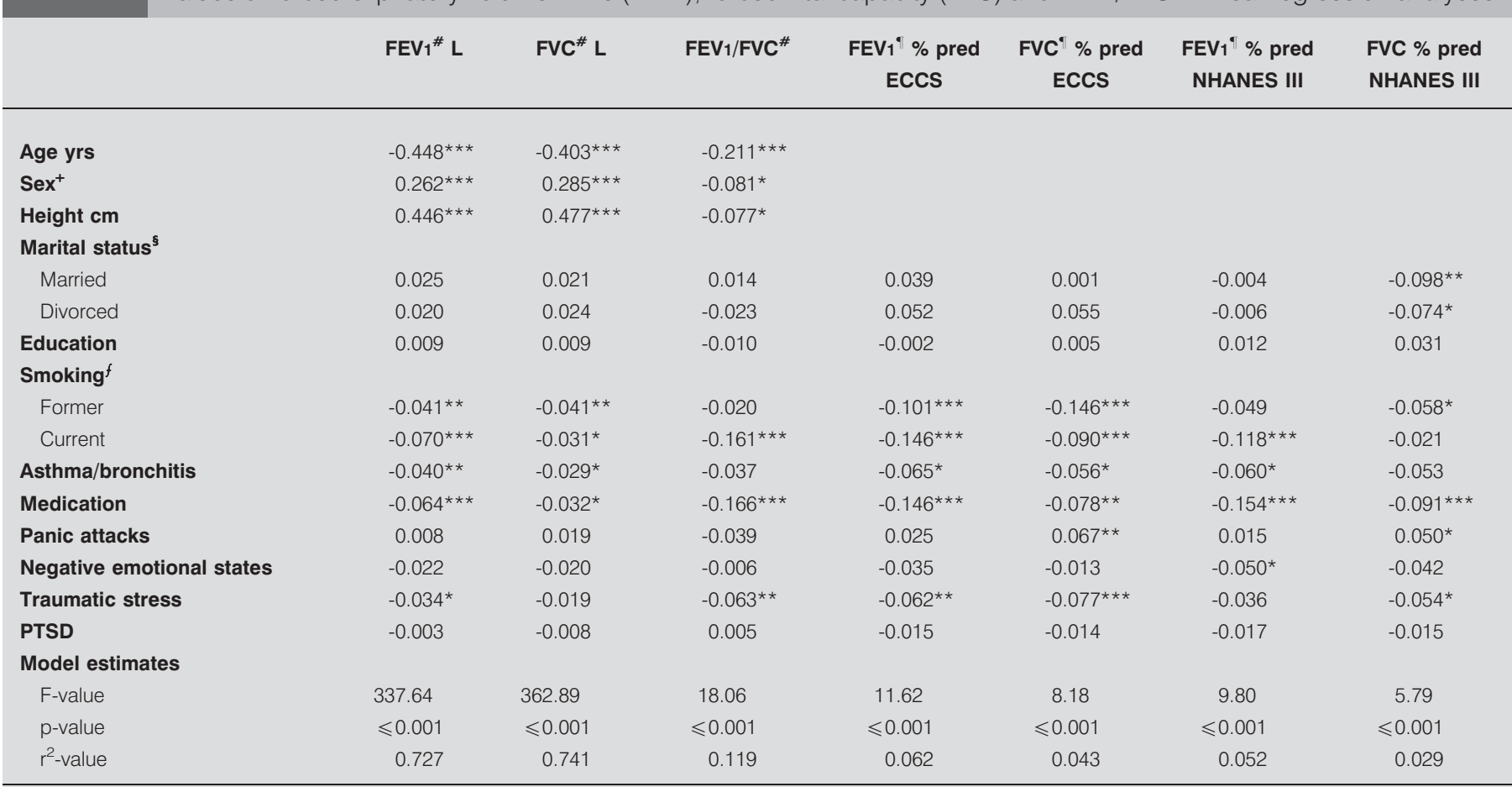

Data are presented as $\beta$. ECCS: European Community for Coal and Steel; NHANES III: Third National Health and Nutrition Examination Survey. \#: linear regression analysis adjusted for age, sex, height, marital status, education, smoking, asthma/bronchitis, medication, panic attacks and negative emotional states; ': linear regression analysis adjusted for marital status, education, smoking, asthma/bronchitis, medication, panic attacks and negative emotional states; ${ }^{+}$: females as reference group; $^{\varsigma}$ : never married as reference group; ${ }^{f}$ : never-smokers as reference group. ${ }^{*}: p \leqslant 0.05 ;{ }^{* *}: p \leqslant 0.01 ;{ }^{* * *}: p \leqslant 0.001$.

With respect to biological mechanisms, chronic traumatic stress and PTSD are characterised by changes in the hypothalamic-pituitary-adrenal axis and the sympathetic-adrenalmedullary system [48]. It was suggested that these alterations lead to a pro-inflammatory state $[1,14,49,50]$. Thus, inflammation may be the link between trauma exposure and PTSD on the one hand and airflow limitation on the other, which itself is associated with inflammatory processes [51].

Our study has some major strengths, including the populationbased design, controlling for potential confounders, exclusion of individuals with cognitive impairment, assessment of traumatic events and PTSD with a psychometrically sound interview [35], as well as the use of spirometric lung function testing. However, some methodological limitations merit discussion. First, because our investigation was cross-sectional, the reported associations do not allow any causal inferences. More specifically, information about the temporal relationship of respiratory symptoms and lung function findings relative to trauma exposure and PTSD onset was not available. However, there is evidence that PTSD-positive subjects do not have a higher risk of physical illness before trauma exposure than those without PTSD [16], suggesting that traumatic experiences are likely to have predated airflow limitation in most cases. Nevertheless, we are in need of prospective studies to confirm this. Secondly, the lifetime prevalence of trauma exposure found in our study (51.6\%) may appear high for a general population sample, thus questioning whether it is representative. However, epidemiological studies in adult community populations have reported lifetime prevalences for traumatic events ranging $20-90 \%$ depending on the definition and assessment of traumatic experiences, the country where the study was conducted and age range of the sample $[30,52]$. Consistent with our results, a study of the adult general population in the Netherlands found that $52.2 \%$ of subjects reported the experience of at least one traumatic event [53]. In contrast, the lifetime prevalence of PTSD in our investigation (1.6\% of the total sample) appears low compared to US figures, e.g. $7.8 \%$ in the National Comorbidity Survey [54], but is very similar to the lifetime prevalence of $1.4 \%$ reported by another German general population study [55]. Again, differences in methodology and socioeconomic background of the study populations can explain divergent results [30]. Thirdly, our general population sample cannot be considered representative for two reasons. The follow-up sample differed from the baseline sample [31], and there were significant sociodemographic and clinical differences between participants performing spirometry and those who did not. Fourthly, panic attacks and negative emotional states were only assessed by a screening instrument, and not by specific measures or structured interviews. Furthermore, the additional assessment of the personality trait negative affectivity, reflecting the tendency to experience negative emotions [56], might have been useful, as it is positively related to elevated symptom reporting [19]. Finally, small numbers of participants with PTSD, and self-reported pulmonary diseases 
and objectively defined airflow limitation may have compromised statistical power, i.e. the precision of risk estimates was low in some instances. Thus, we may have even failed to find "true" associations between PTSD and measures of airflow limitation.

Notwithstanding these caveats, our study, in concert with prior research, strongly indicates an association of traumatic experiences and PTSD with obstruction of the respiratory system. From a clinical point of view, diagnostic and treatment attention should be paid to obstructive pulmonary diseases in traumatised and PTSD-positive subjects both in primary care, and special medical and mental health settings. It was suggested that treatment of PTSD may reduce allostatic load and, thus, positively influences physical health [57]. Prospective studies are needed to further clarify the association between trauma, PTSD and respiratory dysfunction, as well as the beneficial impact of PTSD treatment on pulmonary diseases.

\section{SUPPORT STATEMENT}

The work is part of the Community Medicine Research network (CMR) of the University of Greifswald, Germany, which is funded by the Federal Ministry of Education and Research (grant numbers ZZ9603, 01ZZ0103 and 01ZZ0403), Competence Network Asthma/COPD (FKZ 01GI0881-0888), the Ministry of Cultural Affairs as well as the Social Ministry of the Federal State of Mecklenburg-West Pomerania. The CMR encompasses several research projects which are sharing data of the population-based Study of Health in Pomerania (SHIP; http:// ship.community-medicine.de).

\section{STATEMENT OF INTEREST}

None declared.

\section{REFERENCES}

1 Boscarino JA. Posttraumatic stress disorder and physical illness: results from clinical and epidemiologic studies. Ann NY Acad Sci 2004; 1032: 141-153.

2 Schnurr PP, Green BL, eds. Trauma and Health: Physical Health Consequences of Exposure to Extreme Stress. Washington, American Psychological Association, 2004.

3 Spitzer C, Barnow S, Volzke H, et al. Trauma, posttraumatic stress disorder, and physical illness: findings from the general population. Psychosom Med 2009; 71: 1012-1017.

4 Felitti VJ, Anda RF, Nordenberg D, et al. Relationship of childhood abuse and household dysfunction to many of the leading causes of death in adults. The Adverse Childhood Experiences (ACE) Study. Am J Prev Med 1998; 14: 245-258.

5 Goodwin RD, Wamboldt MZ, Pine DS. Lung disease and internalizing disorders. Is childhood abuse a shared etiologic factor? J Psychosom Res 2003; 55: 215-219.

6 Goodwin RD, Stein MB. Association between childhood trauma and physical disorders among adults in the United States. Psychol Med 2004; 34: 509-520.

7 Scott KM, Von Korff M, Alonso J, et al. Childhood adversity, earlyonset depressive/anxiety disorders, and adult-onset asthma. Psychosom Med 2008; 70: 1035-1043.

8 Brackbill RM, Hadler JL, DiGrande L, et al. Asthma and posttraumatic stress symptoms 5 to 6 years following exposure to the World Trade Center terrorist attack. JAMA 2009; 302: 502-516.

9 Fagan J, Galea S, Ahern J, et al. Relationship of self-reported asthma severity and urgent health care utilization to psychological sequelae of the September 11, 2001 terrorist attacks on the World
Trade Center among New York City area residents. Psychosom Med 2003; 65: 993-996.

10 Farfel M, DiGrande L, Brackbill R, et al. An overview of 9/11 experiences and respiratory and mental health conditions among World Trade Center Health Registry enrollees. J Urban Health 2008; 85: 880-909.

11 Sareen J, Cox BJ, Stein MB, et al. Physical and mental comorbidity, disability, and suicidal behavior associated with posttraumatic stress disorder in a large community sample. Psychosom Med 2007; 69: 242-248.

12 Seng JS, Clark MK, McCarthy AM, et al. PTSD and physical comorbidity among women receiving Medicaid: results from service-use data. J Trauma Stress 2006; 19: 45-56.

13 Dobie DJ, Kivlahan DR, Maynard C, et al. Posttraumatic stress disorder in female veterans: association with self-reported health problems and functional impairment. Arch Intern Med 2004; 164: 394-400.

14 O'Toole BI, Catts SV. Trauma, PTSD, and physical health: an epidemiological study of Australian Vietnam veterans. J Psychosom Res 2008; 64: 33-40.

15 Spiro A 3rd, Hankin CS, Mansell D, et al. Posttraumatic stress disorder and health status: the veterans health study. J Ambul Care Manage 2006; 29: 71-86.

16 Weisberg RB, Bruce SE, Machan JT, et al. Nonpsychiatric illness among primary care patients with trauma histories and posttraumatic stress disorder. Psychiatr Serv 2002; 53: 848-854.

17 Goodwin RD, Fischer ME, Goldberg J. A twin study of posttraumatic stress disorder symptoms and asthma. Am J Respir Crit Care Med 2007; 176: 983-987.

18 Frueh BC, Hamner MB, Cahill SP, et al. Apparent symptom overreporting in combat veterans evaluated for PTSD. Clin Psychol Rev 2000; 20: 853-885.

19 Janssens T, Verleden G, De Peuter S, et al. Inaccurate perception of asthma symptoms: a cognitive-affective framework and implications for asthma treatment. Clin Psychol Rev 2009; 29: 317-327.

20 Ritz T, Steptoe A. Emotion and pulmonary function in asthma: reactivity in the field and relationship with laboratory induction of emotion. Psychosom Med 2000; 62: 808-815.

21 Schnurr PP, Spiro A 3rd, Paris AH. Physician-diagnosed medical disorders in relation to PTSD symptoms in older male military veterans. Health Psychol 2000; 19: 91-97.

22 Beckham JC, Moore SD, Feldman ME, et al. Health status, somatization, and severity of posttraumatic stress disorder in Vietnam combat veterans with posttraumatic stress disorder. Am J Psychiatry 1998; 155: 1565-1569.

23 Schnurr PP, Spiro A 3rd, Aldwin CM, et al. Physical symptom trajectories following trauma exposure: longitudinal findings from the normative aging study. J Nerv Ment Dis 1998; 186: 522-528.

24 Glaser S, Schaper C, Obst A, et al. Impact of different definitions of airflow limitation on the COPD prevalence in a general population. Respiration 2010; 80: 292-300.

25 Pauwels RA, Buist AS, Ma P, et al. Global strategy for the diagnosis, management, and prevention of chronic obstructive pulmonary disease: National Heart, Lung, and Blood Institute and World Health Organization Global Initiative for Chronic Obstructive Lung Disease (GOLD): executive summary. Respir Care 2001; 46: 798-825.

26 Quanjer PH, Tammeling GJ, Cotes JE, et al. Lung volumes and forced ventilatory flows. Report Working Party Standardization of Lung Function Tests, European Community for Steel and Coal. Official Statement of the European Respiratory Society. Eur Respir J Suppl 1993; 16: 5-40.

27 Hankinson JL, Odencrantz JR, Fedan KB. Spirometric reference values from a sample of the general U.S. population. Am J Respir Crit Care Med 1999; 159: 179-187. 
28 Keil U, Cairns V, Doering A, et al. MONICA Project, Region Augsburg, Manual of Operations. Munich, Gesellschaft fuer Strahlenforschung (GSF-Bericht 20/85), 1985.

29 John U, Greiner B, Hensel E, et al. Study of Health In Pomerania (SHIP): a health examination survey in an east German region: objectives and design. Soz Praventivmed 2001; 46: 186-194.

30 Spitzer C, Barnow S, Volzke H, et al. Trauma and posttraumatic stress disorder in the elderly: findings from a German community study. J Clin Psychiatry 2008; 69: 693-700.

31 Haring R, Alte D, Volzke H, et al. Extend recruitment efforts minimize attrition but not necessarily bias. J Clin Epidemiol 2009; 62: 252-260.

32 Glaser S, Friedrich N, Ewert R, et al. Association between serum insulin-like growth factor (IGF) I and IGF binding protein-3 and lung function. J Clin Endocrinol Metab 2009; 94: 2452-2458.

33 Koch B, Schaper C, Ittermann $\mathrm{T}$, et al. Reference values for cardiopulmonary exercise testing in healthy volunteers: the SHIP study. Eur Respir J 2009; 33: 389-397.

34 First MB, Spitzer RL, Gibbon M, et al. Structured Clinical Interview for DSM-IV Axis I Disorders. Washington, American Psychiatric Press, 1997.

35 Elhai JD, Gray MJ, Kashdan TB, et al. Which instruments are most commonly used to assess traumatic event exposure and posttraumatic effects? A survey of traumatic stress professionals. J Trauma Stress 2005; 18: 541-545.

36 Wittchen HU, Hoefler M, Gander F, et al. Screening for mental disorders: performance of the Composite International Diagnostic - Screener (CID-S). Int J Meth Psych Res 1999; 8: 59-70.

37 Folstein MF, Folstein SE, McHugh PR. "Mini-mental state". A practical method for grading the cognitive state of patients for the clinician. J Psychiatr Res 1975; 12: 189-198.

38 Anda RF, Brown DW, Dube SR, et al. Adverse childhood experiences and chronic obstructive pulmonary disease in adults. Am J Prev Med 2008; 34: 396-403.

39 Chinn S, Jarvis D, Burney $\mathrm{P}$, et al. Increase in diagnosed asthma but not in symptoms in the European Community Respiratory Health Survey. Thorax 2004; 59: 646-651.

40 WHO. WHO Collaborating Centre for Drug Statistics Methodology. Geneva, WHO, 2005.

41 Standardization of spirometry-1987 update. Statement of the American Thoracic Society. Am Rev Respir Dis 1987; 136: 1285-1298.

42 Standardization of spirometry-1987 update. Official statement of American Thoracic Society. Respir Care 1987; 32: 1039-1060.

43 MacCallum RC, Zhang S, Preacher KJ, et al. On the practice of dichotomization of quantitative variables. Psychol Methods 2002; 7: $19-40$.
44 Green BL, Kimerling R. Trauma, PTSD, and health status. In Schnurr PP, Green BL, eds. Trauma and Health: Physical Health Consequences of Exposure to Extreme Stress. Washington, American Psychological Association, 2004; pp. 13-42.

45 Schnurr PP, Green BL. Understanding relationships among trauma, posttraumatic stress disorder, and health outcomes In: Schnurr PP, Green BL, eds. Trauma and Health: Physical Health Consequences of Exposure to Extreme Stress. Washington, American Psychological Association, 2004; pp. 150-159.

46 Norman SB, Means-Christensen AJ, Craske MG, et al. Associations between psychological trauma and physical illness in primary care. J Trauma Stress 2006; 19: 461-470.

47 McEwen BS, Seeman T. Protective and damaging effects of mediators of stress. Elaborating and testing the concepts of allostasis and allostatic load. Ann N Y Acad Sci 1999; 896: 30-47.

48 Yehuda R. Biology of posttraumatic stress disorder. J Clin Psychiatry 2000; 61: Suppl. 7, 14-21.

49 Spitzer C, Barnow S, Volzke H, et al. Association of posttraumatic stress disorder with low-grade elevation of C-reactive protein: evidence from the general population. J Psychiatr Res 2010; 44: 15-21.

50 von Kanel R, Hepp U, Kraemer B, et al. Evidence for low-grade systemic proinflammatory activity in patients with posttraumatic stress disorder. J Psychiatr Res 2007; 41: 744-752.

51 Rasmussen F, Mikkelsen D, Hancox RJ, et al. High-sensitive Creactive protein is associated with reduced lung function in young adults. Eur Respir J 2009; 33: 382-388.

52 Breslau N. Epidemiologic studies of trauma, posttraumatic stress disorder, and other psychiatric disorders. Can J Psychiatry 2002; 47: 923-929.

53 Bronner MB, Peek N, Vries M, et al. A community-based survey of posttraumatic stress disorder in the Netherlands. J Trauma Stress 2009; 22: 74-78.

54 Kessler RC, Sonnega A, Bromet E, et al. Posttraumatic stress disorder in the National Comorbidity Survey. Arch Gen Psychiatry 1995; 52: 1048-1060.

55 Hapke U, Schumann A, Rumpf HJ, et al. Post-traumatic stress disorder: the role of trauma, pre-existing psychiatric disorders, and gender. Eur Arch Psychiatry Clin Neurosci 2006; 256: 299-306.

56 Watson D, Pennebaker JW. Health complaints, stress, and distress: exploring the central role of negative affectivity. Psychol Rev 1989; 96: 234-254.

57 Friedman MJ, McEwen BS. Posttraumatic stress disorder, allostatic load, and medical illness. In: Schnurr PP, Green BL, eds. Trauma and Health: Physical Health Consequences of Exposure to Extreme Stress. Washington, American Psychological Association, 2004; pp. 160-189. 